\title{
"COMPARATIVE STUDY OF FNAC, USG, US-FNAC, AND HPE IN DIAGNOSIS OF NECK SWELLINGS"
}

Authors: Sachin Jain (1), Surendra Kumar Gupta(2), Kachnar Varma(3), Rajendra Kumar Gola* (4) Ridhima Malik(4), Sachin Singh(4)

Authors Affiliations: (1) Professor and Head, (2) Associate Professor, (3) Professor, (4) Junior Resident, Junior Resident, Department of ENT \& Head Neck Surgery, M.L.N. Medical College, Prayagraj, Uttar Pradesh, India

\begin{abstract}
Aims: This prospective study was carried out to compare findings of the four procedures namely FNAC (fine-needle aspiration cytology), USG (ultrasonography), US-FNAC (Ultrasound-guided fine-needle aspiration cytology), and HPE (histopathological examination).
\end{abstract}

\section{MATERIAL AND METHODS}

Total 80 cases of different neck swellings were selected from ENT OPD at tertiary care hospital Prayagraj Uttar Pradesh. All cases underwent the preoperative procedure of FNAC,USG, US-FNAC and postoperative HPE for diagnosing the neck swelling. The results of FNAC, USG, US-FNAC were compared and correlated with histopathology findings and conclusions drawn after statistical analysis.

\section{RESULTS}

More than half (56.25\%) of neck swellings were of thyroid swellings. It was observed that sensitivity, specificity, PPV, NPV, accuracy of FNAC of all neck swellings were $85.71 \%, 91.78 \%, 50 \%, 98.53 \%$ ,91.25\% respectively. Sensitivity, specificity, PPV ,NPV , accuracy of all neck swellings of USG were $71.43 \%, 98.63 \%, 83.33 \%, 97.26 \% 96.25$ respectively. Sensitivity ,specificity, PPV ,NPV , accuracy of all neck swellings of US-FNAC were $85.71 \%, 97.26 \%, 75 \%, 98.61 \%, 96.25$ respectively.

\section{CONCLUSION}

The combined use of USG and FNAC give more accuracy for proper diagnosis of neck swelling than FNAC alone. The most accurate diagnosis of neck swelling based on FNAC, USG, and USFNAC also depends upon the experience of cytopathologists and radiologists however the confirmatory diagnosis is given by HPE.

Keywords: FNAC, USG, US-FNAC, HPE, Neck Swellings.

\section{INTRODUCTION}

Neck swelling originates from many structures in the neck like the thyroid gland, salivary glands, lymph nodes, soft tissues, blood vessels, and neural structures. Thyroglossal duct cyst is the most common congenital mass of the midline of the neck1.The prevalence of thyroid nodules has been estimated to be as high as $64 \%$, with the incidence of malignancy ranging from 5 to $10 \%$, depending on the population under study2.FNACas a diagnostic procedure to evaluate head and neck swelling was first used by Martin and Ellis in 19303. It is a cheap, safe, procedure, with fast reporting and requires minimal equipment. It reduces the number of thyroidectomies by approximately $50 \% 4$, roughly doubles the surgical yield of carcinoma, and decreases the overall cost by 25\%5.USG for diagnosis of neck lesions was first introduced in 1966-19676. It is harmless, uses nonionizing radiation, widely available, easy to use, noninvasive, inexpensive, and unaffected by metal artifacts such as dental restorations. It gives information to differentiate solid from cystic lesions. It may alert us of the probabilities of the malignant versus benign nature of neck swellings7.US-FNAC gives information about the location of swelling and the relationship to 
adjacent anatomical structures. It carries several benefits, including reduction of multiple physician visits, assessment by a physician familiar with neck anatomy, as well as the potentially decreasing wait time for surgery8.TheHPE report was taken as a confirmatory and final diagnostic procedure in all the cases and compared with the FNAC, USG, and US-FNAC diagnosis.

\section{METHODS}

The present prospective study was carried out in the department of ENT and Head \& Neck Surgery in collaboration with the department of pathology and radiology in tertiary care hospital Pryagraj U.P. for a duration of one year from August 2019 to July 2020.Patients with ages between 10-60 years irrespective of gender with clinically palpable neck swelling were included in the study while inflammatory and metastatic neck swelling were excluded from the study. After making a clinical diagnosis based on history and clinical examination, all 80 patients were subjected to undergo FNAC of the neck swelling in the Department of Pathology, followed by USG neck and US- FNAC of the neck swelling in the Department of Radiology. Routine blood investigations and TFT (thyroid function test)in cases of thyroid swellings were done in all neck swellings. Tissue specimens obtained after the surgical procedure were sent for HPE, in the Department of Pathology. The diagnosis was ultimately confirmed by HPE of the neck swelling. A battery of statistical tests of sensitivity, specificity, PPV (positive predictive value), NPV (negative predictive value), and accuracy was calculated for FNAC, USG, and US-FNAC with HPE.

\section{RESULTS}

A total of 80 patients with various neck swellings was enrolled in the study, among them 13(16.25\%) were male patients and $67(83.75 \%)$ were female patients with a male to female ratio of 1:5.The youngest patient was 15 years old and the eldest patient was 60years old. The most common age group with various neck swellings in this study was $31-40$ years. Majority of patients (75; 93.75\%) presented with swelling over the neck with no other symptoms. The largest swelling was (18x10) $\mathrm{cm}$ in size and the smallest was $(2 \times 2) \mathrm{cm}$ in size. The most common (31; 38.75\%) location of neck swellings was midline. The majority (71; 88.75\%) of neck swellings were firm in consistency and mobile (78; 97.50\%). According to clinical diagnosis, the most common diagnosis was thyroid swelling (45; 56.25\%), followed by parotid gland swellings (18; $22.50 \%), 2(2.50 \%)$ submandibular gland swelling, and $15(18.75 \%)$ were other neck swellings. In 15, other neck swellings' most common clinical diagnosis was cervical lymphadenopathy $(7 ; 8.75 \%)$ followed by cervical swellings $(6 ; 7.50 \%)$ and $1(1.25 \%)$ was submental swelling, and 1 (1.25\%) was submandibular swelling (Table 1).

Table 1.: Distribution of patients based on clinical diagnosis

\begin{tabular}{|l|c|c|}
\hline Clinical diagnosis & $\begin{array}{c}\text { Number of } \\
\text { patients }\end{array}$ & Percentage \\
\hline Thyroid swellings & 45 & 56.25 \\
\hline Parotid gland swellings & 18 & 22.50 \\
\hline Submandibular gland swellings & 2 & 2.50 \\
\hline Other neck swellings & 7 & 8.75 \\
Cervical lymphadenopathy & 6 & 7.50 \\
Cervical swelling & 1 & 1.25 \\
Submental swelling & 1 & 1.25 \\
Submandibular swelling & 80 & 100.00 \\
\hline Total & & \\
\hline
\end{tabular}

According to FNAC diagnosis majority (68; 85\%) were benign neck swellings, followed by suspicious (8;10\%) and $4(5 \%)$ were malignant. In 68 benign neck swellings, the most common was the thyroid (40;50\%),followed by the salivary gland $(16 ; 20 \%)$ and $12(15 \%)$ were other neck swellings. In benign neck swellings, the most common histopathological finding was colloid goiter (fig-1a) (30;37.50\%) followed by pleomorphic adenoma parotid (13;16.25\%) and $4(5 \%)$ were granulomatous lymphadenitis. In suspicious neck swellings, a majority (3;3.75\%) were the lymphoproliferative disorder (fig-1b) of lymph node followed by 2(2.50\%) were thyroid neoplasm, 2(2.50\%) were follicular neoplasm, and 1 was parotid neoplasm. In malignant neck 
swellings, 1 (1.25\%) was papillary carcinoma thyroid,1(1.25\%) malignancy of parotid, and $2(2.50 \%)$ metastatic SCC (squamous cell carcinoma) parotid.

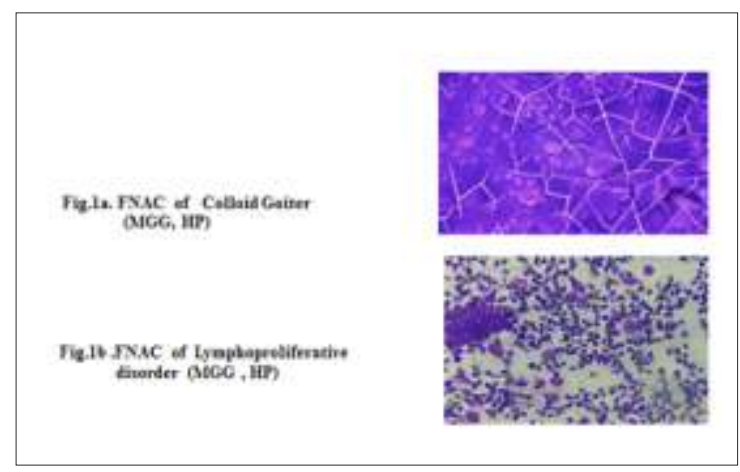

Based on USG diagnosis, 74(92.50\%) neck swellings were found benign, followed by $4(5 \%)$ suspicious and 2(2.25\%) were malignant. In benign neck swellings, a majority (43;53.75\%) were thyroid followed by the salivary gland $(19 ; 23.75 \%)$, and $12(15 \%)$ were other neck swellings. In benign neck swellings, the most common diagnosis was colloid goiter $(19 ; 23.75 \%)$, solitary thyroid nodule was $12(15 \%)$ (Fig 2a).pleomorphic adenoma parotid(Fig 2b). was15(18.75\%), 3(3.75\%) were thyroglossal cyst, and 3(3.375\%). tubercular cervical lymph node. In suspicious neck swellings, 1(1.25\% ) was thyroid neoplasm,1(1.25\%) right parotid mass and 2(2.50\%)were enlarged lymph node mass. In malignant neck swellings, $1(1.25 \%$ ) was malignancy of thyroid and $1(1.25 \%)$ malignancy of parotid.

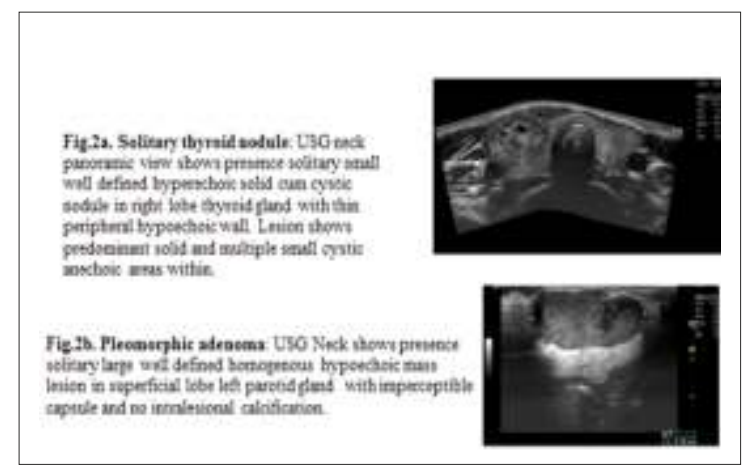

Based on US-FNAC diagnosis $72(90 \%)$ were benign neck swellings, 4 (5\%) were suspicious and $4(5 \%)$ were malignant. In benign neck swellings, the majority (41;51.25\%)were thyroid followed by the salivary gland (18;22.50\%),and $13(16.25 \%)$ were others' neck swellings. In benign neck swellings, the most common diagnosis was colloid goiter $(34 ; 42.50 \%)$ followed by pleomorphic adenoma parotid (Fig.3a)(14;17.50\%)and 5(6.25\%) were granulomatous Iymphadenitis (Fig.3b).In suspicious neck swellings, 2(2.50\%) were follicular neoplasm and 2(2.50\%) were the lymphoproliferative disorder of the lymph node. In malignant neck swellings,1(1.25\%) was papillary carcinoma thyroid, $1(1.25 \%)$ anaplastic carcinoma of the thyroid, and $2(2.50 \%)$ was malignancy of parotid.

On HPE 73(91.25\%) were benign neck swellings

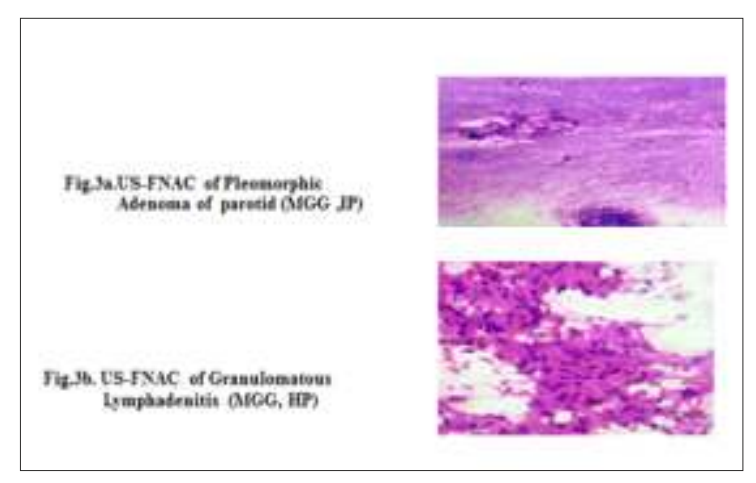

and 7 (8.75\%) were malignant. In benign neck swellings most common diagnosis was colloid goiter 32(40\%), the second most common was pleomorphic adenoma of parotid 14 (17.50\%). In the malignant category, 2(2.50\%) were papillary carcinoma thyroid (Fig.4a) (most common malignancy of thyroid), 1(1.25\%) was anaplastic carcinoma thyroid, 2(2.50\%) was mucoepidermoid carcinoma parotid (Fig.4b), $1(1.25 \%)$ Hodgkin lymphoma, and $1(1.25 \%)$ was non-Hodgkin lymphoma.

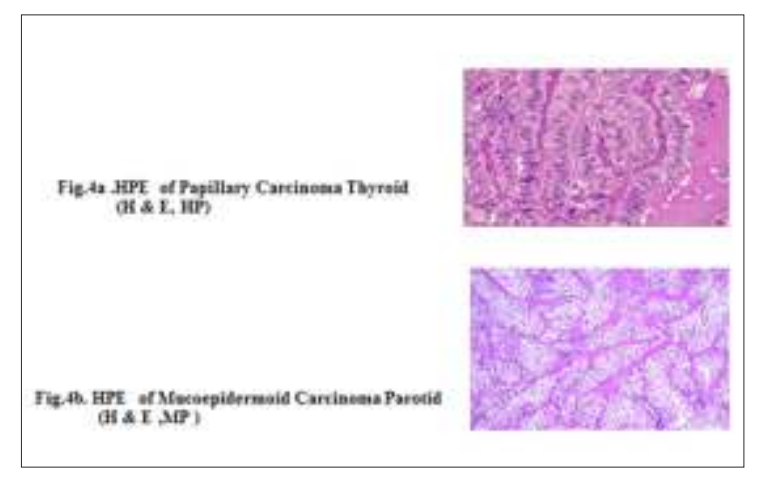


The distribution of 80 cases diagnosed by FNAC, USG, US-FNAC, and HPE is given below (Table.2). Table.2 Distribution of 80 cases diagnosed by FNAC, USG, US-FNAC, and HPE

\begin{tabular}{|l|c|c|c|c|}
\hline Procedure & Benign & Malignant & Suspicious & Total \\
\hline FNAC & 68 & 4 & 8 & 80 \\
\hline USG & 74 & 2 & 4 & 80 \\
\hline US-FNAC & 72 & 4 & 4 & 80 \\
\hline HPE & 73 & 7 & - & 80 \\
\hline
\end{tabular}

For statistical analysis cases on FNAC, USG and US-FNAC were classified as positive (malignant plus suspicious cases) and negative (benign cases).

One benign case (FN; false negative) on FNAC proved to be malignant on HPE. Two malignant cases (FP; false positive) were proved to be benign on HPE. Eight cases of suspicious malignant neck swellings proved to be four benign (FP) and four malignant (TP; true positive) on HPE. Two malignant (TP) cases proved to be malignant on HPE .and sixty-seven benign cases ( $T N$ : true negative) on FNAC proved to be benign on HPE.(Table.3).

TP and TN cases were $6(2+4)$ and 67 respectively.

FP and FN cases were 6(2+4) and 1 respectively.

Table.3 Distribution of cases diagnosed by FNAC and correlation with HPE

\begin{tabular}{|l|c|c|c|}
\hline FNAC (n=80) & \multicolumn{3}{|l|}{ HPE } \\
\hline \multirow{2}{*}{ Positive } & Malignant & Benign & Total \\
\cline { 2 - 4 } & $6(\mathrm{TP})$ & $6(\mathrm{FP})$ & 12 \\
\hline Negative & $1(\mathrm{FN})$ & $67(\mathrm{TN})$ & 68 \\
\hline Total & 7 & 73 & 80 \\
\hline
\end{tabular}

Sensitivity of FNAC of all swellings $=T P X$ $100 / T P+F N=85.71 \%$

Specificity of FNAC of all swellings $=T N X$ $100 / \mathrm{TN}+\mathrm{FP}=91.78 \%$

PPV of FNAC of all swellings $=\mathrm{TP} \times 100 / \mathrm{TP}+\mathrm{FP}=$ $50 \%$

NPV of FNAC of all swellings $=\mathrm{TN} \times 100 / \mathrm{TN}+\mathrm{FN}=$ 98.53\%

Accuracy of FNAC of all swellings $=(\mathrm{TP}+\mathrm{TN}) \mathrm{X}$ 100/TOTAL $=91.25 \%$
Two benign cases (FN) on USG turned out to be malignant on HPE. Four cases of suspicious malignant neck swellings proved to be 1 benign (FP) and 3 malignant (TP)on HPE. Two malignant cases (TP) proved to be malignant on HP and seventy-two benign cases (TN) on USG proved to be benign on HPE.(Table.4).

TP and TN cases were $5(2+3)$ and 72 respectively $\mathrm{FP}$ and $\mathrm{FN}$ cases were 1 and 2 respectively

Table.4 Distribution of cases diagnosed by USG and correlation with HPE

\begin{tabular}{|l|l|l|l|}
\hline USG (n=80) & \multicolumn{3}{|l|}{ HPE } \\
\hline \multirow{2}{*}{ Positive } & Malignant & Benign & Total \\
\cline { 2 - 4 } & $5(\mathrm{TP})$ & $1(\mathrm{FP})$ & 6 \\
\hline Negative & $2(\mathrm{FN})$ & $72(\mathrm{TN})$ & 74 \\
\hline Total & 7 & 73 & 80 \\
\hline
\end{tabular}

Sensitivity of USG of all neck swellings $=71.43 \%$

Specificity of USG of all neck swellings $=98.63 \%$

PPV of USG of all neck swellings $=83.33 \%$

NPV of USG of all neck swellings $=97.29 \%$

Accuracy of USG of all neck swellings $=96.25 \%$

One benign case(FN) on US-FNAC proved to be malignant on HPE. Four cases of suspicious malignant neck swellings proved to be two benign (FP) and two malignant (TP) on HPE. Four malignant cases(TP) proved to be malignant on HPE and seventy-one benign cases (TN) proved to be benign on HPE.(Table.5).

TP and TN cases were 6(4+2) and 71 respectively. $\mathrm{FP}$ and $\mathrm{FN}$ cases were 2 and 1 respectively

Table.5 Distribution of cases diagnosed by USFNAC and correlation with HPE

\begin{tabular}{|l|c|c|c|}
\hline \multicolumn{1}{|c|}{ US-FNAC $(\mathbf{n}=\mathbf{8 0})$} & \multicolumn{3}{|c|}{ HPE } \\
\hline \multirow{2}{*}{ Positive } & Malignant & Benign & Total \\
\cline { 2 - 4 } & $6(\mathrm{TP})$ & $2(\mathrm{FP})$ & 8 \\
\hline Negative & $1(\mathrm{FN})$ & $71(\mathrm{TN})$ & 72 \\
\hline Total & 7 & 73 & 80 \\
\hline
\end{tabular}

Sensitivity of US-FNAC of all neck swellings = $85.71 \%$

Specificity US-FNAC of all neck swellings = $97.26 \%$

PPV of US-FNAC of all neck swellings $=75 \%$ NPV of US-FNAC of all neck swellings $=98.61 \%$ 
Accuracy of US-FNAC of all neck swellings = 96.25\%

It was observed sensitivity, specificity, PPV ,NPV , accuracy of FNAC were $85.71 \%, 91.78 \%, 50 \%$, $98.53 \%, 91.25 \%$ respectively. Sensitivity, specificity, PPV ,NPV , accuracy of USG were $71.43 \%, 98.63 \%, 83.33 \%, 97.26 \% 96.25$ respectively. Sensitivity ,specificity, PPV ,NPV , accuracy of US-FNAC were $85.71 \%, 97.26 \%, 75 \%$ ,98.61\%, 96.25 respectively

\section{DISCUSSION}

Among the several methods available today for evaluating the neck swellings FNAC, USG, and US-FNAC form the preliminary array of modalities because of their availability, simple to perform, and minimally invasive. They play an important role in evaluating the neck swellings.

In total 80 patients were studied, the youngest patient was 15 years old and the eldest was 60 years old. The mean age of patients was 32.67 years, which is close to the study by Gupta et al. 9 study in which the mean age was 38.72 years. The most common neck swelling was thyroid swelling ( $45 ; 56.25 \%$ ) and the most common age group was $31-40$ years $(29 ; 36.50 \%)$ which is close to a study done by Garud P et al.10in which the most common neck swelling was thyroid swelling and the most common age group was 31-40 years. Male to female ratio of 1:5 which is similar to a study done by Garud $\mathrm{P}$ et al.10 with male to female ratio of 1:4.The female majority suggests that hormonal factors may be involved and there can be biological changes during pregnancy which may increase the risk as shown in a study done by Nagarkaret al.11.

FNAC is the cornerstone procedure for the evaluation of neck swellings because its use in recent years has resulted in a significant decrease in the number of surgeries being performed. In the majority of cases, it distinguishes between benign and malignant lesions quite effectively preoperatively so it is also considered as the preoperative screening method of choice. In our study for the diagnosis of neck swellings sensitivity, specificity PPV, NPV, and accuracy of FNAC were $85.71 \%$, $91.78 \%, 50 \%, 98.53 \%, 91.25 \%$ respectively which is similar to Tilak et al12study in which the sensitivity, specificity, and accuracy of FNAC were $90.91 \%, 93.18 \%, 92.73 \%$ respectively. In Nautiyal S et al. 13 study sensitivity (78.9\%)of FNAC was lower while specificity $(96.29 \%)$ and accuracy (95\%) were close to our study. In Garud $P$ et al.10studysensitivity, specificity, and accuracy of FNAC were $90.32 \%, 86.66 \%$,and $89.43 \%$ respectively which are close to our study. The difference in the sensitivity, specificity, and accuracy between our study and others may be due to differences in the method of aspiration of the neck swelling and some pitfalls in FNAC of the thyroid such as adequacy of the specimen (quantitative and qualitative), the accuracy of the specimen (non homogeneity of needle placement), the accuracy of cytopathologic interpretation, cysts (difficulties with degenerative nodules),follicular lesions (benign vs. Malignant), Hurthle cell lesions (benign vs. Malignant) and lymphocytic lesions (lymphocytic thyroiditis vs. Lymphoma)

USG has become the first line of imaging modality for evaluation of the neck swellings due to excellent visualization of the internal parenchyma. Its sensitivity for detecting small swellings, calcification, septations, and cysts is very high and also improves the evaluation of patients with different types of neck swellings. In our study for the diagnosis of neck swellings, sensitivity, specificity, PPV, NPV, and accuracy of USG were $71.43 \%, 98.63 \%, 83.33 \%, 97.29 \%$ $96.25 \%$ respectively while in Farah Naazet al.14study accuracy of USG was $86 \%$ which is lower than our study. In a study by Alshoabi et al.15sensitivity of USG was $98.38 \%$ which was higher than our study and specificity was $71.42 \%$ which is lower than that of our study. The difference in the sensitivity, specificity, and accuracy of USG between our study and others may be due to associated with equipment, examination skills, anatomy, interpretation, and extrathyroidal abnormalities as mentioned by Choi et al.16. 
In the diagnosis of neck swellings, sensitivity, specificity, PPV, NPV, the accuracy of US-FNAC were 85.71\%, 97.26\%, 83.33\%, 97.29\%, 97.50\% respectively while Hee Woo Cho et al.17 study sensitivity of US-FNAC was 76\% which is lower than our study and specificity of US-FNAC was $100 \%$ which is higher than that of our study. In Chakravarthy, et al.18study sensitivity, specificity, and accuracy of US-FNAC were $83.9 \%, 76.3 \%$, and $81 \%$ respectively which is lower than that of our study while Muhammad Ayoob Jat19 study sensitivity, specificity, and accuracy of US-FNAC were $87.5 \%, 95.3 \%$, and $92 \%$ respectively which is lower than that of our study.

\section{CONCLUSION}

Neck swelling requires an accurate diagnosis for an effective treatment plan. FNAC, USG, and USFNAC give minimally invasive sensitive, specific, and accurate diagnosis of neck swelling for proper treatment. In our study Comparative study between FNAC, USG, and US-FNAC in the diagnosis of neck swellings, FNAC is more sensitive as compared to USG however USFNAC more accurate than FNAC but the difference in sensitivity and specificity between USG, FNAC, and US-FNAC are not significant. The combined use of USG and FNAC gives more accuracy for proper diagnosis of neck swelling than FNAC alone. The most accurate diagnosis of neck swelling based on FNAC, USG, and USFNAC also depends upon the experience of cytopathologists and radiologists however the confirmatory diagnosis is given by HPE.

\section{REFERENCES}

1. Allard RH. The thyroglossal cyst. Head Neck Surg. 1982;5:134-46.

2. Ogilvie JB, Piatigorsky EJ, Clark OH. Current status of fine needle aspiration for thyroid nodules. Adv Surg. 2006;40:223-238

3. Martin HE, Ellis EB. Biopsy by needle puncture and aspiration. Annals of surgery. 1930 Aug;92(2):169.

4. Korun N, Aşci C, Yilmazlar T, Duman $H$, Zorluoglu A, Tuncel E, Ertürk E, Yerci O. Total thyroidectomy or lobectomy in benign nodular disease of the thyroid: changing trends in surgery. International surgery. 1997 Oct 1;82(4):417-9.

5. Mazzaferri EL. Management of a solitary thyroid nodule. New England Journal of Medicine. 1993 Feb 25;328(8):553-9.

6. Fujimoto YA, Oka A, Omoto R, Hirose M. Ultrasound scanning of the thyroid gland as a new diagnostic approach. Ultrasonics. 1967 Jul 1;5(3):177-80.

7. Goyal A, Tiwari RS, Desai AA. Diagnostic role of ultrasonography in neck swellings. Indian Journal of Otolaryngology and Head and Neck Surgery. 1999 Oct 1;51(4):67-71.

8. Seiberling KA, Dutra JC, Gunn J. Ultrasound-guided fine needle aspiration biopsy of thyroid nodules performed in the office. The Laryngoscope. 2008 Feb;118(2):228-3.

9. Gupta M, Gupta S, Gupta VB. Correlation of fine needle aspiration cytology with histopathology in the diagnosis of solitary thyroid nodule. Journal of thyroid research. 2010 Apr 18;2010:1-5.

10. Garud P et al.Clinicopathological evaluation of benign neck masses with emphasis on correlation of preoperative ultrasound and cytology withpostoperative histopathology in tertiary care hospital.Int J Otorhinolaryngol Head Neck Surg. 2019 Jul;5(4):946-953.

11. Nagarkar R, Roy S, Akheel M, Palwe V, Kulkarni N,Pandit P. Incidence of Thyroid Disorders in India: An Institutional Retrospective Analysis. Int J Dent Med Spec 2015;2:19-23.

12. Tilak V, Dhaded AV, Jain R. Fine needle aspiration cytology of head and neck masses. Indian J PatholMicrobiol. 2002;45(1):23-9.

13. Nautiyal S, Jain A, Tiwari S, Ashutosh K. Fine needle aspiration cytology accuracy in diagnosis of thyroid lesions: a comparative study with histopathological examination of thyroid swellings. International Journal of Otorhinolaryngology and Head and Neck 
Surgery.2018 Jul;4(4):1002.

14. Naaz F, Choudhry UA, Qaiyum HA. Role of ultrasonography in the diagnosis of neck mass.Journal of Medical \& Allied Sciences. 2018;8(2):66-72.

15. Alshoabi SA, Binnuhaid AA. Diagnostic accuracy of ultrasonography versus fine-needle-aspiration cytology for predicting benign thyroid lesions. Pak J Med Sci. 2019;35(3):630-635.

16. SeonHyeong Choi, Eun-Kyung Kim, Soo Jin Kim, Jin Young Kwak. Thyroid Ultrasonography: Pitfalls and Techniques. Korean J Radiol 2014;15(2):267-276.

17. Cho HW, Kim J, Choi J, Choi HS, Kim ES, Kim $\mathrm{SH}$, Choi EC. Sonographically guided fineneedle aspiration biopsy of major salivary gland masses: a review of 245 cases.American Journal of Roentgenology. 2011 May;196(5):1160-3.

18. Chakravarthy NS, Chandramohan A, Prabhu AJ, Gowri M, Mannam P, Shyamkumar NK, Naik D, Cherian AJ, Thomas N, Paul MJ, Abraham D. Ultrasound-guided fine-needle aspiration cytology along with clinical and radiological features in predicting thyroid malignancy in nodules $\geq 1 \mathrm{~cm}$. Indian journal of endocrinology and metabolism. 2018 Sep;22(5):597.

19. Jat MA. Comparison of surgeon-performed ultrasound-guided fine needle aspiration cytology with histopathological diagnosis of thyroid nodules.Pak J Med Sci. 2019;35(4):1003-1007.

\footnotetext{
*Corresponding author:

Dr. Rajendra Kumar Gola Junior Resident, Department of ENT \& Head Neck Surgery, M.L.N. Medical College, Prayagraj, Uttar Pradesh, India Phone (or Mobile) No.:+917982358326

Email:drrajendragola@gmail.com
} 